\title{
Superfluid and magnetic states of an ultracold Bose gas with synthetic three-dimensional spin-orbit coupling in an optical lattice
}

\author{
Dan-Wei Zhang, ${ }^{1,2}$ Ji-Pei Chen, ${ }^{3}$ Chuan-Jia Shan, ${ }^{1,4}$ Z. D. Wang, ${ }^{2, *}$ and Shi-Liang Zhu ${ }^{1,3, \dagger}$ \\ ${ }^{1}$ Laboratory of Quantum Engineering and Quantum Materials, SPTE, South China Normal University, Guangzhou 510006, China \\ ${ }^{2}$ Department of Physics and Center of Theoretical and Computational Physics, The University of Hong Kong, \\ Pokfulam Road, Hong Kong, China \\ ${ }^{3}$ Laboratory of Solid State Microstructures and Department of Physics, Nanjing University, Nanjing 210093, China \\ ${ }^{4}$ College of Physics and Electronic Science, Hubei Normal University, Huangshi 435002, China
}

(Received 18 January 2013; published 8 July 2013)

\begin{abstract}
We study ultracold bosonic atoms with synthetic three-dimensional spin-orbit (SO) coupling in a cubic optical lattice. In the superfluidity phase, the lowest energy band exhibits one, two, or four pairs of degenerate single-particle ground states depending on the SO-coupling strengths, which can give rise to condensate states with spin stripes for weak atomic interactions. In the deep Mott-insulator regime, the effective spin Hamiltonian of the system combines three-dimensional Heisenberg exchange interactions, anisotropy interactions, and Dzyaloshinskii-Moriya interactions. Based on Monte Carlo simulations, we numerically demonstrate that the resulting Hamiltonian with an additional Zeeman field has a rich phase diagram with spiral, stripe, vortex crystal, and especially Skyrmion crystal spin textures in each $x y$-plane layer. The obtained Skyrmion crystals can be tunable with square and hexagonal symmetries in a columnar manner along the $z$ axis, and moreover are stable against the interlayer spin-spin interactions in a large parameter region.
\end{abstract}

DOI: 10.1103/PhysRevA.88.013612

PACS number(s): 67.85.-d, 05.30.Jp, 71.70.Ej, 37.10.Jk

\section{INTRODUCTION}

Spin-orbit (SO) coupling plays an important role in condensed matter physics, especially in the newly discovered quantum spin Hall effects and the related topological orders $[1,2]$. Recent theoretical proposals $[3,4]$ and experimental realization [5-8] of non-Abelian gauge fields in ultracold atoms with the optical dressing technique open another door to explore SO coupling in controllable systems. The bulk gases of weakly interacting bosons with synthetic two-dimensional (2D) Rashba SO coupling in homogenous cases and in trapping potentials have been widely studied and theoretically shown to exhibit exotic many-body ground states [9], some of which have no direct analog in solid-state systems [10-13]. For example, an SO-coupled spinor condensate will spontaneously develop a plane-wave phase or spin-stripe structure depending on the weak interaction energy $[10,11]$; and in the presence of strong trapping potentials, it will exhibit half-quantum vortex states and Skyrmion patterns [11-13].

Recently, physics of an SO-coupled bosonic gas loaded in a $2 \mathrm{D}$ optical lattice $(\mathrm{OL})$ has attracted considerable interest [14-23]. This system can be described by an extended twocomponent Bose-Hubbard (BH) model [24-26], in which the SO coupling can significantly affect the quantum phase transition from a superfluid to a Mott insulator (MI) $[15,16,20]$. More interestingly, the effective spin Hamiltonian of the system in the deep MI regime contains the so-called DzyaloshinskiiMoriya (DM) interaction term [27], which comes from the SO coupling and may lead to some novel magnetic phases [16-19], such as Skyrmion crystals [16]. However, the corresponding three-dimensional (3D) system is yet to be explored.

\footnotetext{
*zwang@hku.hk

†slzhu@scnu.edu.cn
}

On the other hand, the 3D analog of SO coupling in cold atoms has been proposed to be experimentally realized by using optical dressing schemes [28,29] and by exploiting laser-assisted tunneling [30] in OLs [31,32]. The 3D SO couplings are less explored in contrast to the standard 2D Rashba and Dresselhaus ones in the context of condensed matter physics [28], but is now attracting more and more interest [2,33-35] for investigating 3D topological insulators [2], topological superfluidity [34], Weyl semimetals [33], and spherical-SO-coupling-induced Bardeen-Cooper-Schreiffer to Bose-Einstein condensate (BEC) crossover [35]. Very recently, several pieces of theoretical work study the ground state of a weakly interacting two-component BEC with 3D SO-coupling in the continuum [36-38]. It is shown that the density distribution of the ground-state BEC can also exhibit the interesting Skyrmion structure, which is moreover a 3D counterpart characterized by a 3D topological winding number $[36,37]$. Some other schemes have also been proposed to create Skyrmions in multicomponent BECs in the absence of synthetic SO couplings and OLs [39]. So, it would be worthwhile to search the stable Skyrmion (crystals) in an OL system with 3D SO-coupled bosons.

In this paper, we investigate ultracold bosons with synthetic 3D SO coupling in a cubic OL. We first look into the weakly interacting superfluidity case. In this case, the lowest energy band exhibits one, two, or four pairs of degenerate single-particle ground states related to the SO coupling, and each pair contains opposite wave vectors with values depending on the SO-coupling strengths. This can give rise to condensate states with spin stripes for the weak atomic interactions. We then focus on the deep MI regime with one atom per lattice and derive the effective spin-spin interaction Hamiltonian of the system. The spin Hamiltonian is a combination of three-dimensional Heisenberg exchange interactions, anisotropy interactions, and DM interactions. Based on Monte 
Carlo (MC) simulations, we numerically demonstrate that the resulting Hamiltonian with an additional Zeeman field has a rich classical phase diagram with spiral, stripe, vortex crystal, and especially Skyrmion crystal spin textures in each $x y$-plane layer. We find that the obtained Skyrmion crystals can be square or hexagonal symmetries with experimentally tunable parameters by varying laser-atom interactions. Moreover, the Skyrmion crystals in a columnar manner of extending along the $z$ axis are stable against the interlayer spin interactions within a large parameter region. This cold atom system with high controllability in the effective spin interactions may provide an ideal platform to further study exotic quantum spin models and find new phases of matter.

The paper is organized as follows. The next section (Sec. II) introduces an extended $\mathrm{BH}$ model which describes cold bosons with synthetic 3D SO coupling in a cubic OL. In Sec. III, we briefly analyze the single-particle energy band and the properties of the weakly interacting superfluidity phase. In Sec. IV, we derive the spin Hamiltonian of the system in the MI regime, and present its rich classical phase diagram with interesting spin configurations. In particular, we study the profiles and stability of the Skyrmion crystals in the system. A brief discussion and short conclusion are finally given in Sec. V.

\section{MODEL}

Let us consider an atomic gas of pseudospin-1/2 ultracold bosons loaded into a 3D cubic optical lattice with synthetic SO coupling. The single-particle Hamiltonian of the system is written as

$$
\hat{H}_{0}=\frac{\hat{\mathbf{p}}^{2}}{2 m}+\kappa_{x} \sigma_{x} \hat{p}_{x}+\kappa_{y} \sigma_{y} \hat{p}_{y}+\kappa_{z} \sigma_{z} \hat{p}_{z}+V(x, y, z),
$$

where $m$ is the atomic mass, $\hat{\mathbf{p}}$ is the momentum operator, $\kappa_{\eta}$ with $\eta=x, y, z$ is, respectively, the strength of SO coupling along the $\eta$ axis, and $\sigma_{x, y, z}$ are the three Pauli matrices. Here the cubic optical lattice $V(x, y, z)=\sum_{\eta} V_{\eta} \sin ^{2}\left(k_{0} \eta\right)$ is formed by three standing-wave laser beams with the same wave number $k_{0}$. Thus the lattice spacing is $a=\pi / k_{0}$. Hamiltonian (1) can be rewritten as $\hat{H}_{0}=(\hat{\mathbf{p}}-\mathbf{A})^{2} / 2 m+V$ up to a constant, where the non-Abelian gauge potential $\mathbf{A}=$ $-m\left(\kappa_{x} \sigma_{x}, \kappa_{y} \sigma_{y}, \kappa_{z} \sigma_{z}\right)$. This corresponds to a 3D SO coupling.

We consider the system in the tight-binding regime, which is reachable in realistic experiments [25,26]. Under this condition, in the presence of such a non-Abelian gauge field, the bosons can be described by an extended single-band $\mathrm{BH}$ Hamiltonian in terms of Peierls substitution [16,17]:

$$
\mathcal{H}=-\sum_{\mathbf{i}, \hat{\eta}}\left[t_{\eta} \hat{a}_{\mathbf{i}, \sigma}^{\dagger} \mathcal{R}_{\eta}^{\sigma \sigma^{\prime}} \hat{a}_{\mathbf{i}+\hat{\eta}, \sigma^{\prime}}+\text { H.c. }\right]+V_{\mathrm{int}},
$$

where $\hat{a}_{\mathbf{i}, \sigma}^{\dagger}\left(\hat{a}_{\mathbf{i}, \sigma}\right)$ creates (annihilates) a spin- $\sigma(\sigma=\uparrow, \downarrow)$ boson at site $\mathbf{i}$. The first term in Hamiltonian (2) describes atomic hopping between neighbor lattice sites, with $t_{\eta}$ representing the overall hopping amplitude in the absence of synthetic SO coupling. The $2 \times 2$ matrix $\mathcal{R}_{\eta} \equiv \exp \left(-\frac{i}{\hbar} A_{\eta} a\right)$ is the Peierls substitution along direction $\hat{\eta}$ with respect to the gauge potential. We rewrite $\mathcal{R}_{\eta}=\exp \left(i \theta_{\eta} \sigma_{\eta}\right)=\cos \theta_{\eta} \mathbf{1}+i \sigma_{\eta} \sin \theta_{\eta}$ with dimensionless SO-coupling strength $\theta_{\eta}=\pi m \kappa_{\eta} / \hbar k_{0}$. The diagonal and off-diagonal terms in the matrix respectively refer to the spin-conserving hopping and spin-flip hopping due to the SO coupling in the $x y$ plane. The second term in Hamiltonian (2) denotes the atomic repulsive interactions, which is given by

$$
V_{\mathrm{int}}=\frac{1}{2} \sum_{\mathbf{i}, \sigma \sigma^{\prime}} U_{\sigma \sigma^{\prime}} \hat{a}_{\mathbf{i}, \sigma}^{\dagger} \hat{a}_{\mathbf{i}, \sigma^{\prime}}^{\dagger} \hat{a}_{\mathbf{i}, \sigma^{\prime}} \hat{a}_{\mathbf{i}, \sigma},
$$

where $U_{\sigma \sigma^{\prime}}$ is the interaction strength between spins $\sigma$ and $\sigma^{\prime}$. The atomic interactions are almost spin independent in experiments in the absence of Feshbach resonances $[5,6]$, and hence we assume $U_{\uparrow \uparrow}=U_{\downarrow \downarrow} \equiv U$ and $U_{\uparrow \downarrow}=U_{\downarrow \uparrow}=\alpha U$ with $\alpha \approx 1$. In fact, the slight difference between the intraspecies and interspecies interaction strengths will help to select the degenerate many-body ground states of the system in the weak interacting superfluidity phase.

This extended 3D BH model also exhibits the superfluidity and the MI phases for the weak and strong atomic interactions compared with the hopping energy [25,26], respectively. The quantum phase transitions between them are affected by the SO coupling in a similar manner as that in the $2 \mathrm{D}$ cases $[15,16,20]$. Thus we just consider the system in the two interaction limits in this work, and focus on the effects of synthetic 3D SO coupling in the superfluidity and the MI phases.

\section{SUPERFLUIDITY STATES}

In this section, we consider the weakly interacting superfluidity phase. In this regime, the hopping term dominates in Hamiltonian (2). We first look into the hopping Hamiltonian $\mathcal{H}_{T}=\mathcal{H}-V_{\text {int }}$ to obtain the energy band of the system, and then briefly discuss the effects of weak atomic interactions. The corresponding Hamiltonian in the momentum space can be written as

$$
\mathcal{H}_{\mathbf{k}}=\sum_{\mathbf{k}}\left(\hat{a}_{\mathbf{k}, \uparrow}^{\dagger} \hat{a}_{\mathbf{k}, \downarrow}^{\dagger}\right) \hat{H}_{\mathbf{k}}\left(\begin{array}{l}
\hat{a}_{\mathbf{k}, \uparrow} \\
\hat{a}_{\mathbf{k}, \downarrow}
\end{array}\right) .
$$

By using spatial Fourier transformations on $\mathcal{H}_{T}$, we can obtain $\hat{H}_{\mathbf{k}}=H_{k x}+H_{k y}+H_{k z}$ with $H_{k \eta}=-2 t_{\eta} \cos \theta_{\eta} \cos \left(k_{\eta} a\right)$ $\mathbf{1}+2 t_{\eta} \sin \theta_{\eta} \sin \left(k_{\eta} a\right) \sigma_{\eta}$. Diagonalizing $\hat{H}_{\mathbf{k}}$ yields the energy structure of the system in the vanishing interaction limit:

$E_{\mathbf{k}}^{ \pm}=-2 \sum_{\eta} t_{\eta} \cos \theta_{\eta} \cos \left(k_{\eta} a\right) \pm 2 \sqrt{\sum_{\eta} t_{\eta}^{2} \sin ^{2} \theta_{\eta} \sin ^{2}\left(k_{\eta} a\right)}$.

The lowest energy states in the resulting lower Bloch band $E_{\mathbf{k}}^{-}$present candidates for the many-body ground state of the bosonic gas with weak interatomic interactions. The Bloch momentum of these states denoted by $\mathbf{k}_{0} \equiv\left(k_{0}^{x}, k_{0}^{y}, k_{0}^{z}\right)$ can be directly obtained by solving the equation $\left.\partial_{\mathbf{k}}\left(E_{\mathbf{k}}^{-}\right)\right|_{\mathbf{k}=\mathbf{k}_{0}}=0$ for minimizing $E_{\mathbf{k}}^{-}$with the specific parameters $t_{\eta}$ and $\theta_{\eta}$. We find that there are possibly one, two, or four pairs of degenerate minima in the lowest energy band for different values of $t_{\eta}$ and $\theta_{\eta}$ in the case of nonvanishing SO coupling. Each pair come as a time-reversed partner with opposite wave vectors. For simplicity, we focus on the isotropic tunneling with $t_{x}=t_{y}=t_{z}$; the degeneracy of the ground states can be split into three cases by the configuration of the SO coupling [35]. The first case is that the SO coupling is anisotropic along all three dimensions. We then have a single pair of degenerate 
minima. If it is anisotropic along two dimensions-whether we have one pair, or two pairs, depending on the configuration of the SO coupling-it is prolate or oblate [35]. Finally, the case of maximum symmetry with fully isotropic SO coupling gives four pairs of degenerate minima. For example, when $\theta_{x}=$ $\theta_{y}<\theta_{z}$ (the prolate case), two degenerate minima locate at $\mathbf{k}_{0}=\left(0,0, \pm \theta_{z} / a\right)$; when $\theta_{x}=\theta_{y}>\theta_{z}$ (the oblate case), four degenerate minima locate at $\left( \pm \xi_{1} / a, \pm \xi_{1} / a, 0\right)$ with $\tan \xi_{1}=$ $\tan \theta_{x} / \sqrt{2}$; when $\theta_{x}=\theta_{y}=\theta_{z}$, eight degenerate minima locate at $\left( \pm \xi_{2} / a, \pm \xi_{2} / a, \pm \xi_{2} / a\right)$ with $\tan \xi_{2}=\tan \theta_{x} / \sqrt{3}$. This is in sharp contrast to the continuum case where the rotationally symmetric dispersion has an infinite ground-state degeneracy forming an SO sphere [28]. Owing to the reduction of degeneracy, the Bose condensates with synthetic 3D SO coupling in an OL would be more robust against quantum fluctuations than their bulk counterparts.

For the weakly interacting cases (i.e., $U \ll t_{\eta}$ ) within the Gross-Pitaevskill (GP) approximation, the analysis of the ground state (condensate) wave function in this system is in parallel to those of the counterparts in 2D OLs [16], as well as in 2D and 3D continuum cases [10,37]. Hence we just present the conclusions here without detailed calculations. The condensate wave function can be written as a superposition of all possible single-particle (plane-wave) wave functions of the lowest Bloch states discussed above, and the corresponding superposition coefficients are determined by minimizing the mean-field GP interaction (i.e., density-density interaction) energy $[10,37]$. The GP interaction energy can be divided into the spin-independent and spin-dependent parts. The spinindependent term yields the same Hartree-Fock energy for any different selection of superposition coefficients, but the spin-dependent one with respect to $\alpha$ selects the coefficients for minimizing itself. For $\alpha<1$, only one of the lowest degenerate single-particle states (whose numbers can be two, four, or eight) is occupied, and thus the condensate wave function is a plane-wave state with a finite momentum. On the other hand, for $\alpha>1$, one of the paired degenerate states (whose numbers can be one, two, or four) are occupied with equal superposition coefficients, giving rise to the condensate states with spin-stripe density distribution $[10,11]$. The structure of the spin stripe is dependent on the vector $\mathbf{k}_{0}$ and hence is tunable by the synthetic SO coupling. We note that these ground states are still degenerate except in the case of stripe states with only two degenerate minima in the Bloch band. To further remove this accidental degeneracy, one should consider quantum fluctuations [40].

\section{SPIN MODEL AND MAGNETIC STATES IN MOTT-INSULATOR REGIME}

\section{A. Effective spin Hamiltonian}

In this section, we consider the system in the MI phase. We are interested in the MI regime with $U \gg t_{\eta}$ and nearly unit atom per lattice site. In this case, the atoms are localized in individual lattices and the nearest-neighbor hopping can be treated as a perturbation, leading to an effective spin Hamiltonian [41]. To obtain the spin Hamiltonian of the system, one can begin with a two-site problem [42]. In the zero order, the system is described by the interaction
Hamiltonian (3), and the ground-state manifold for the twosite problem with one atom in each site composes four degenerate zero-energy states $\{|\uparrow ; \uparrow\rangle,|\uparrow ; \downarrow\rangle,|\downarrow ; \uparrow\rangle,|\downarrow ; \downarrow\rangle\}$. Here we have assumed uniform in-site energy and chosen it as the energy base. The exchange of two atoms in different sites does not require energy, and hence the single atom hopping should be eliminated in the second order with respect to the ratio $t_{\eta} / U$. In this progress, there are six excited states $\{|\uparrow \downarrow ; 0\rangle,|0 ; \uparrow \downarrow\rangle,|\uparrow \uparrow ; 0\rangle,|0 ; \uparrow \uparrow\rangle,|\downarrow \downarrow ; 0\rangle,|0 ; \downarrow \downarrow\rangle\}$ with an energy $U$. The hopping perturbation described by $\mathcal{H}_{\mathrm{T}}$ couples the ground-state manifold and the excited-state one. The resulting effective Hamiltonian up to the second order of perturbation reads $[41,42]$

$$
\left(H_{\mathrm{eff}}\right)_{\beta v}=-\sum_{\gamma} \frac{\left(H_{\mathrm{T}}\right)_{\beta \gamma}\left(H_{\mathrm{T}}\right)_{\gamma \nu}}{E_{\gamma}-\left(E_{\beta}+E_{v}\right) / 2},
$$

where $\beta$ and $v$ label the four states in the ground-state manifold and $\gamma$ labels the six excited ones.

After obtaining the two-site effective Hamiltonian from Eq. (6), it is straightforward to extend it to the lattice counterpart by introducing nearest-neighbor hopping in the whole lattice. It is convenient to write the lattice effective Hamiltonian in terms of isospin operators $\vec{S}_{\mathbf{i}}=\left(S_{\mathbf{i}}^{x}, S_{\mathbf{i}}^{y}, S_{\mathbf{i}}^{z}\right)$ with $S_{\mathbf{i}}^{x}=\frac{1}{2}\left(\hat{a}_{\mathbf{i}, \uparrow}^{\dagger} \hat{a}_{\mathbf{i}, \downarrow}+\hat{a}_{\mathbf{i}, \downarrow}^{\dagger} \hat{a}_{\mathbf{i}, \uparrow}\right), S_{\mathbf{i}}^{y}=-\frac{i}{2}\left(\hat{a}_{\mathbf{i}, \uparrow}^{\dagger} \hat{a}_{\mathbf{i}, \downarrow}-\hat{a}_{\mathbf{i}, \downarrow}^{\dagger} \hat{a}_{\mathbf{i}, \uparrow}\right)$, and $S_{\mathbf{i}}^{z}=\frac{1}{2}\left(\hat{a}_{\mathbf{i}, \uparrow}^{\dagger} \hat{a}_{\mathbf{i}, \uparrow}-\hat{a}_{\mathbf{i}, \downarrow}^{\dagger} \hat{a}_{\mathbf{i}, \downarrow}\right)$. The resulting spin Hamiltonian of this $3 \mathrm{D}$ system in the deep MI region is then given by

$$
\begin{aligned}
\mathcal{H}_{s}= & -\sum_{\mathbf{i}} \vec{S}_{\mathbf{i}} \cdot\left(J_{x} \vec{S}_{\mathbf{i}+\hat{x}}+J_{y} \vec{S}_{\mathbf{i}+\hat{y}}+J_{z} \vec{S}_{\mathbf{i}+\hat{z}}\right) \\
& -\sum_{\mathbf{i}}\left(K_{x} S_{\mathbf{i}}^{x} S_{\mathbf{i}+\hat{x}}^{x}+K_{y} S_{\mathbf{i}}^{y} S_{\mathbf{i}+\hat{y}}^{y}+K_{z} S_{\mathbf{i}}^{z} S_{\mathbf{i}+\hat{z}}^{z}\right) \\
& -\sum_{\mathbf{i}}\left(D_{x} \vec{S}_{\mathbf{i}} \times \vec{S}_{\mathbf{i}+\hat{x}} \cdot \hat{x}+D_{y} \vec{S}_{\mathbf{i}} \times \vec{S}_{\mathbf{i}+\hat{y}} \cdot \hat{y}\right. \\
& \left.+D_{z} \vec{S}_{\mathbf{i}} \times \vec{S}_{\mathbf{i}+\hat{z}} \cdot \hat{z}\right),
\end{aligned}
$$

where $\quad J_{\eta}=\frac{4 t_{\eta}^{2}}{U} \cos \left(2 \theta_{\eta}\right), \quad K_{\eta}=\frac{8 t_{\eta}^{2}}{U} \sin ^{2} \theta_{\eta}, \quad$ and $\quad D_{\eta}=$ $\frac{4 t_{\eta}^{2}}{U} \sin \left(2 \theta_{\eta}\right)$ are spin interaction strengths. Hamiltonian (7) describes a generally anisotropic 3D spin-spin interaction system. It combines the Heisenberg exchange interaction as the first term, the anisotropy interaction as the second term, and 3D DM spin interaction as the last term. Note that all the spin interaction strengths in these terms are dependent on the laser beams which generate the OL and the SO coupling, and hence they are tunable in experiments.

To proceed further, we introduce an effective Zeeman term to Hamiltonian (7), leading to the total spin Hamiltonian

$$
\mathcal{H}_{s}^{T}=\mathcal{H}_{s}-h_{z} \sum_{\mathbf{i}} S_{\mathbf{i}}^{z}
$$

This Zeeman term can be easily achieved by applying an additional external field to the pseudospin-1/2 atoms. For the pseudospin states that are usually two atomic hyperfine states, the external field can be simply a real magnetic field $[42,43]$. If the pseudospin states are dressed states, one can use an appropriately designed laser field to generate it [44]. Thus $h_{z}$ is also a tunable parameter. We assume that the strength of the Zeeman field here is $h_{z} \ll U$ but is comparable with $t_{\eta}^{2} / U$. 
Under this condition, the parameters in Hamiltonian (7) are approximately unchanged.

\section{B. Numerical results from Monte Carlo simulations}

Below we explore the low-temperature phase diagrams and ground states of the effective spin Hamiltonian $\mathcal{H}_{s}^{T}$ [see Eq. (8)], and we also intend to find stable Skyrmionic spin textures via MC simulations. In this classical approximation we treat the spins $\vec{S}_{\mathbf{i}}$ as classical unit vectors and aim to find the spin configurations $\left\{\vec{S}_{\mathrm{i}}\right\}$ for minimizing the energy. We note that the classical MC simulation has been used to explore the phase diagrams of several kinds of spin models, such as the Heisenberg model with DM interactions in the context of solids $[45,47,48]$. While this method may not be used to determine the precise phase boundaries and to search for some phases driven by quantum fluctuations, it can be an efficient tool to determine different possible phases, especially when there are no degeneracies on the classical level. For example, the classical MC results in Ref. [16] are consistent with those by the variational analysis in Ref. [19]. Thus one can expect that the classification of ground states in our MC simulations could generally survive in a variational approach, but a full quantum treatment would be needed to have a deeper understanding of the quantum spin model (8), which is beyond the present work.

For simplicity, we assume isotropic parameters in the $x y$ plane, i.e., $t_{x}=t_{y} \equiv t$ and $\theta_{x}=\theta_{y} \equiv \theta$, and take $4 t^{2} / U$ as energy unit hereafter, such that $J_{x}=J_{y}=\cos (2 \theta), K_{x}=K_{y}=$ $2 \sin ^{2} \theta$ and $D_{x}=D_{y}=\sin (2 \theta)$. We also assume $t_{z}=\lambda_{1} t$ and $\theta_{z}=\lambda_{2} \theta$, such that $J_{z}=\lambda_{1}^{2} \cos \left(2 \lambda_{2} \theta\right), K_{z}=2 \lambda_{1}^{2} \sin ^{2}\left(\lambda_{2} \theta\right)$, and $D_{z}=\lambda_{1}^{2} \sin \left(2 \lambda_{2} \theta\right)$. Calculations were mostly carried out for an $18 \times 18 \times 18$ lattice with periodic boundary conditions. Metropolis MC algorithm [49] was used throughout the calculations with $5 \times 10^{6}$ (and $5 \times 10^{5}$ for an $x y$ layer with $18 \times 18$ lattice) sampling steps at each annealing process with fixed low temperature $T=0.005$ (in units of $4 t^{2} / U$ ). Some checks on each $x y$ plane with sites $36 \times 36$ were performed to ensure consistency. The numerical results were also confirmed to be nearly the same for the open and periodic boundary conditions.

\section{Phase diagram of the reduced $2 D$ layer model with $t_{z}=0$}

We first consider the case of $t_{z} \simeq 0$ in the Hamiltonian (8), which can be realized by increasing the intensity of the lasers that generate the periodic lattices along the $\hat{z}$ axis and freeze the atomic motions in this direction. In this case, the $3 \mathrm{D}$ system is equivalent to a collection of independent 2D plane layers along the $\hat{z}$ axis, and thus the spin configurations in each layer are always the same in the ground states. We have confirmed this point in our numerical simulations. So, we can first look into a single layer and figure out the phase diagram of Hamiltonian (8) with $t_{z}=0$. In this limit, the spin Hamiltonian is similar to those in the previous work $[16,17]$ without the additional Zeeman term. We have checked that in this case our results are consistent with those in Refs. $[16,17]$ when the parameter regions are overlapped, i.e., $h_{z}=0, t_{x}=t_{y}$, and $\theta_{x}=\theta_{y}$.

We obtain the classical ground-state phase diagram of the spin Hamiltonian $\mathcal{H}_{s}^{T}$ with $t_{z}=0$ as shown in Fig. 1. Apart from the conventional ferromagnetic (FM) and antiferromagnetic (AFM) phases, there are four unconventional phases

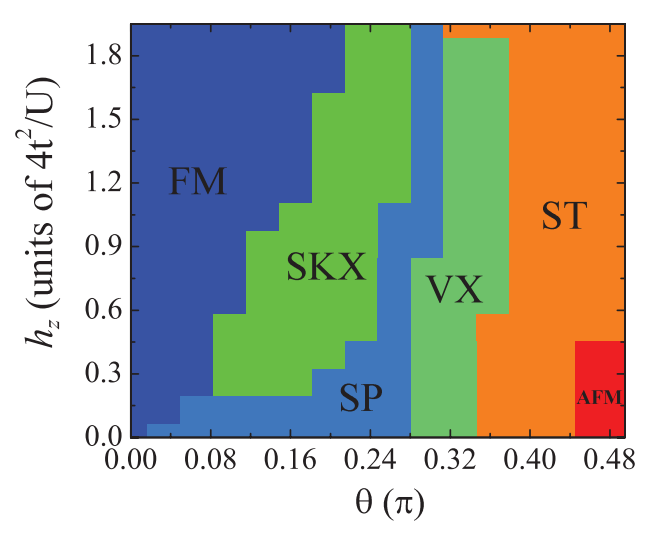

FIG. 1. (Color online) Classical phase diagram of the reduced 2D-layer version of the spin Hamiltonian $\mathcal{H}_{s}^{T}$ with $t_{z}=0$, obtained from Monte Carlo simulations. This is related to the bosons loaded in the OL in the deep MI regime. The abbreviations are as follows: FM. ferromagnetic phase; AFM. antiferromagnetic phase; SP, spiral phase; ST, stripe phase; VX, vortex-crystal phase; and SKX, Skyrmion crystal phase. Their definitions are in the text. Same typical spin configurations of unusual phases (SP, ST, SKX, and VX) are shown in Figs. 2 and 3.

having interesting spin configurations in the $x y$ plane: spiral (SP), stripe (ST), vortex crystal (VX), and Skyrmion crystal (SKX). Here FM denotes a ferromagnet with the spins being aligned along one direction (along the $\hat{z}$ axis in our case due to the Zeeman field), and the corresponding AFM is an antiferromagnet with neighboring spins pointing in opposite directions (along the $\pm \hat{z}$ axis in this paper). SP denotes a coplanar ground state with the spins having a spiral wave in its configuration, whose spatial periodicity is more than two sites. ST denotes another coplanar ground state, with spins being separated by periodically spaced domain walls. The case shown in Fig. 2(c) is a ferromagnetic stripe phase characterized by a single wave vector along the $\hat{x}$ axis. VX denotes a crystal state of vortices, with the spins wound clockwise or counterclockwise around each plaquette in the $x y$ plane. Finally, SKX is a crystal state of Skyrmions, where the spins align in a VX shape with nonzero Skyrmion density given by Eq. (9) below. The presented phase diagram shows a rich interplay between different magnetic orders, and the parameter region of the six phases can be found in Fig. 1. We should note that their boundaries between different phases may be unprecise in the level of quantum phases, however, this classical approach is efficient to determine possible phases [16-18].

The spin textures in the four unconventional magnetic phases have nontrivial structures and can be characterized by their spin structure factors $S_{\mathbf{k}}^{\perp} \equiv\left|\sum_{\mathbf{i}} \vec{S}_{\mathbf{i}}^{\perp} e^{i \vec{k} \cdot \vec{r}_{\mathbf{i}}}\right|^{2}$ with $\vec{S}_{\mathbf{i}}^{\perp}=\left(S_{\mathbf{i}}^{x}, S_{\mathbf{i}}^{y}, 0\right)$. Figures 2(a)-2(d) show some typical spin configurations in the spiral, vortex-crystal, and stripe phases, and Fig. 2(e) shows their spin structure factors $S_{\mathbf{k}}^{\perp}$ in the momentum space (i.e., $k_{x}-k_{y}$ plane), with the spots denoting the peaks of the spin structure factors. In Fig. 2(e), $S_{\mathbf{k}}^{\perp}$ exhibits a peak at $(0,0.125 \pi)$, corresponding to the spins spiraling along the $y$ axis with the wave number $0.125 \pi$ as shown in Fig. 2(a). Similarly, the spins forming a vortex-crystal configuration in Fig. 2(b) has $S_{\mathbf{k}}^{\perp}$ peaks at $(0.75 \pi, 0)$ and $(0,0.75 \pi)$, and the stripe spin configuration in Fig. 2(c) has a single peak at $(\pi, 0)$, 

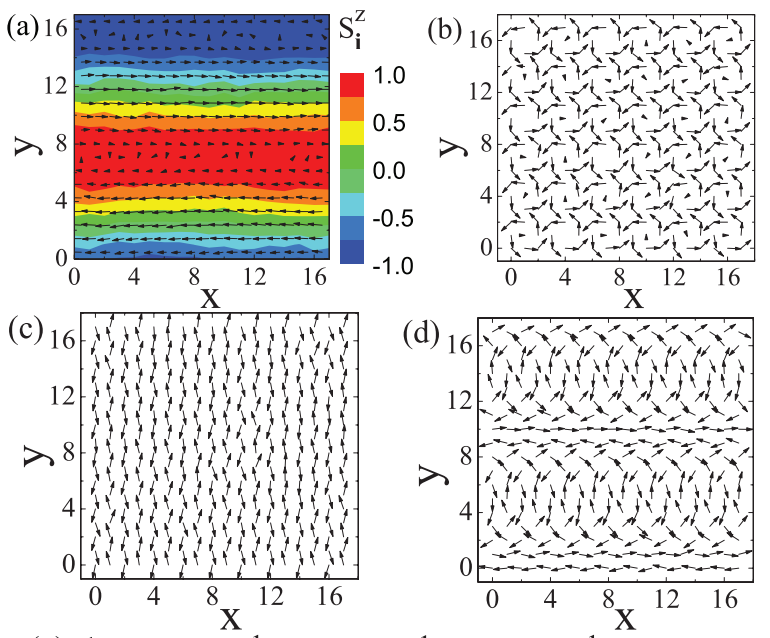

(d) 16
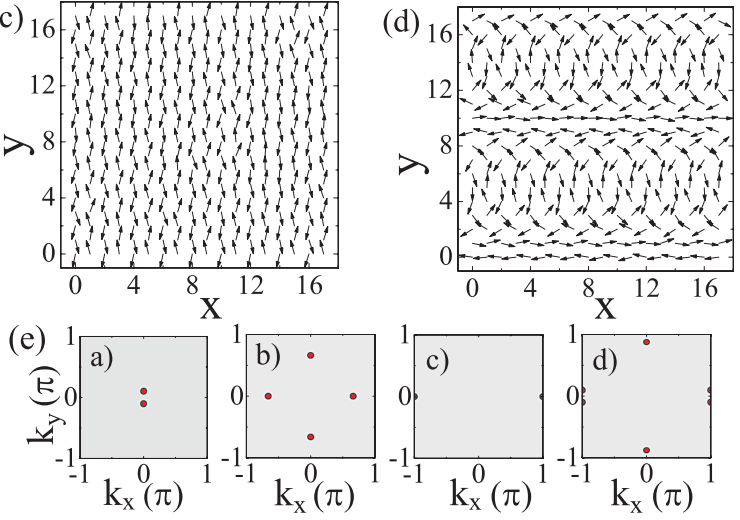

FIG. 2. (Color online) The spin configurations (distribution of $\vec{S}_{\mathbf{i}}$ ) in the $x y$ plane with $z=0$ : (a) $\operatorname{SP}\left(\theta=0.104, h_{z}=0\right)$; (b) $\operatorname{VX}(\theta=1$, $\left.h_{z}=0\right)$; (c) ST $\left(\theta=1.24, h_{z}=0\right)$; and (d) ST with complicated structure $\left(\theta=1.56, h_{z}=2.34\right)$, which is not in the phase diagram. (e) The corresponding spin structure factors $S_{\mathbf{k}}^{\perp}$ (see the text) of the spin configurations in (a)-(d) are from left to right, respectively.

which means that the spins are staggered along the $x$ axis but are parallel along the $y$ axis. We also find that the ground states may exhibit more complicated stripe spin textures outside the parameter region of the phase diagram, with an example being shown in Fig. 2(d).

The Skyrmion crystal phase in the phase diagram, referring to the phase where the spins presenting an array of 2D Skyrmions (see Fig. 3), has a large parameter region when varying the SO-coupling strength $\theta$ and the Zeeman field $h_{z}$. The array of Skyrmions is anisotropy in general, and can present square or hexagonal-symmetric patterns for proper SO-coupling strengths. Figures 3(a) and 3(b) show the typical spin configurations in the Skyrmion crystal phase with square and hexagonal structures, respectively. The corresponding spin structure factors $S_{\mathbf{k}}^{\perp}$ shown in Figs. 3(c) and 3(d) also reflect their symmetries in the momentum space. We note that the hexagonal Skyrmion crystal state in fact exhibits a triangular lattice structure of Skyrmions in the real space since $S_{i}^{z}$ is taken in account [see Fig. 3(b)]. However, we still refer to it as hexagonal in order to be consistent with its hexagonal Bragg pattern in the Fourier analysis [see Fig. 3(d)].

Before ending this part, we analyze the energies of the obtained spin states. For the reduced 2D system here, the energy functional $E\left[\vec{S}_{\mathbf{i}}\right]$ is given by the Hamiltonian (8) with $J_{x, y}=\cos (2 \theta), K_{x, y}=2 \sin ^{2} \theta, D_{x, y}=\sin (2 \theta)$, and $J_{z}=D_{z}=K_{z}=0$. A classic spin state can be parametrized by $\vec{S}_{\mathbf{i}}=S\left(\cos \gamma_{\mathbf{i}} \sin \varphi_{\mathbf{i}}, \sin \gamma_{\mathbf{i}} \sin \varphi_{\mathbf{i}}, \cos \varphi_{\mathbf{i}}\right)$ with $S^{2}=1$ and $\mathbf{i} \doteq\left(x_{i}, y_{i}\right)$. For the $\hat{z}$-axis ferromagnetic and antiferromagnetic spin states in our case, the energy densities defined as the energy per spin are $E_{\mathrm{FM}}=-E_{\mathrm{AFM}}=-h_{z}-2 \cos (2 \theta)$ (here $h_{z}>0$ and $0<\theta<\pi / 2$ ). For the spiral and stripe spin states
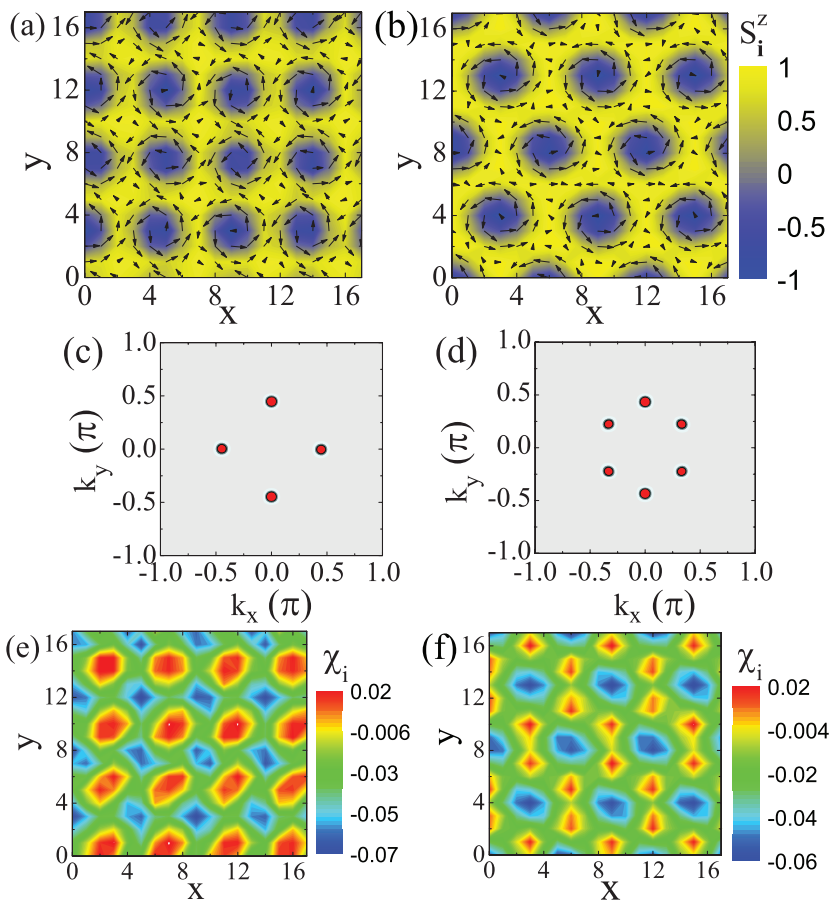

FIG. 3. (Color online) Properties of the 2D Skyrmion crystals. Typical spin configurations in the Skyrmion crystal phase with (a) square and (b) hexagonal symmetry, and the color scale shows the magnitude of out-of-plane component $S_{z}$. (c) and (d) are the corresponding spin structure factors $S_{\mathbf{k}}^{\perp}$ of the spin configurations in (a) and (d), respectively. (e) and (f) are the local density $\chi_{\mathbf{i}}$ (see the text) of the square and the hexagonal Skyrmion crystals, respectively. The parameters are $\theta=0.726$ and $h_{z}=0.910$ for the square Skyrmion crystals; $\theta=0.592$ and $h_{z}=0.756$ for the hexagonal Skyrmion crystals.

shown in Figs. 2(a) and 2(c), based on their spin structures in the momentum space, one can write down their spin configurations as $\vec{S}_{\mathbf{i}, \mathbf{S P}}=\left(\sin \left[k_{\mathrm{SP}} y_{i}\right], 0, \cos \left[k_{\mathrm{SP}} y_{i}\right]\right)$ with $k_{\mathrm{SP}}=0.125 \pi$, and $\vec{S}_{\mathbf{i}, \mathbf{S T}}=\left[(-1)^{x_{i}+y_{i}} \cos \left(k_{\mathrm{ST}} x_{i}+\phi\right), \sin \left(k_{\mathrm{ST}} x_{i}+\phi\right), 0\right]$ with $k_{\mathrm{ST}}=\pi$ and $\phi=0.44 \pi$ being a phase angle. Here we have dropped an irrelevant overall phase. Direct calculations yield the energy densities of the two spin states $E_{\mathrm{SP}}=$ $-\cos (2 \theta)\left(1+\cos k_{\mathrm{SP}}\right)-\sin (2 \theta) \sin k_{\mathrm{SP}}-\sin ^{2} \theta$, and $E_{\mathrm{ST}}=$ $-2 \sin ^{2} \theta$. Here $E_{\mathrm{ST}}$ is independent of $\phi$, which indicates that the stripe spin states in our system with different phase angles are degenerate on the classical level. For the crystal states of vortices and Skyrmions shown in Figs. 2(b), 3(a), and $3(\mathrm{~b})$, which contain more than one spiral wave, it is very hard to give an expression of their spin configurations [45]. For example, one has to replace the local constraint $\vec{S}_{\mathbf{i}}^{2}=1$ followed in our numerical simulations and previous analysis by a global one (i.e., $\left\langle\vec{S}_{\mathbf{i}}^{2}\right\rangle=1$ ) in approximately writing down the spin configuration of the square Skyrmion crystal as $\vec{S}_{\mathbf{i}, \mathbf{S}-\mathrm{SKX}} \propto\left(\sin \left[k_{\mathrm{SKX}} y_{i}\right], \cos \left[k_{\mathrm{SKX}} x_{i}\right], \sin \left[k_{\mathrm{SKX}} x_{i}\right]+\right.$ $\cos \left[k_{\mathrm{SKX}} y_{i}\right]$ ) [45], with the wave number $k_{\mathrm{SKX}}=0.5 \pi$ as shown in Fig. 3(c). However, this approach will lead to a considerable deviation in estimating the energy density of these spin states [45]. Therefore, we numerically compute their energy densities by directly using the obtained spin configurations in Figs. 2(b), 3(a), and 3(b). For the parameters 
$\theta=0.726$ and $h_{z}=0.91$, we get the energy density of the vortex crystal state $E_{\mathrm{VX}}=-1.18$, the square Skyrmion crystal state $E_{\mathrm{S} \text {-SKX }}=-1.77$, and the hexagonal Skyrmion crystal state $E_{\mathrm{H}-\mathrm{SKX}}=-1.73$. One can check that $E_{\mathrm{S}-\mathrm{SKX}}$ is the smallest among all six spin states for the parameters, which indicates that the square Skyrmion crystal state is indeed the classical ground state in this case. For $\theta=0.592$ and $h_{z}=0.756$, we get $E_{\mathrm{VX}}=-0.99, E_{\mathrm{S}-\mathrm{SKX}}=-1.82$, and $E_{\mathrm{H}-\mathrm{SKX}}=-1.86$, so the hexagonal Skyrmion crystal state is the classical ground state in this case.

\section{2D Skyrmion crystals and effects of Zeeman fields}

The 2D Skyrmion crystals obtained in this model are distinguished from those in Ref. [16] not only in their spin configurations but also in the mechanism. The $2 \mathrm{D}$ model in Ref. [16] contains no Zeeman field, however, the Zeeman field is crucial here. As seen from the phase diagram, there are no Skyrmion crystal states without the Zeeman field; they should be generated from the spiral waves with the help of a Zeeman field, while the spiral waves are formed via the competition between the DM interactions and the Heisenberg exchange interactions. In the presence of isotropic DM interactions ( $D_{\eta}$ is independent of $\eta$ ), the spiral spins can give way to the Skyrmion crystal spins in energy for a sufficiently strong Zeeman field or anisotropic spin interactions [45,46]. Because all the spin interactions in our system are interdependent with respect to the SO-coupling strengths [for example, $K_{\eta}$ is larger than $D_{\eta}$ when $\theta_{\eta}>\pi / 4$ in the Hamiltonian (7)], the Skyrmion crystal states can be stabilized only by the Zeeman field (see Fig. 1). The presence of Skyrmion crystal spins in Ref. [16] without a Zeeman field is due to the anisotropic DM interactions, which can increase the effective anisotropic spin interactions. With an increase of Zeeman energy in the SKX regime, some Skyrmion crystal spins begin to melt into the ferromagnetic spins when the Zeeman term becomes dominant over the DM term, and finally all of them realign as a ferromagnet along the $\hat{z}$ axis over a critical value, which is not shown in the phase diagram.

Such Skyrmion crystals have been explored by numerical calculations and in experiments in chiral magnet materials $[45,47,48]$, but the controllability in the materials $[47,48]$ is low in contrast to that in the cold atom system. This system is clean and the parameters, such as $\theta$ and $h_{z}$, are widely tunable via adjusting laser-atom interations $[25,26]$. So, the density of Skyrmions and the symmetry of the Skyrmion crystal can be well controlled by varying the two parameters. For instance, increasing the Zeeman field can lead to an increase of the Skyrmion density up to certain levels, and varying the SOcoupling strength can change the distribution of Skyrmions, from generally anisotropy to square or hexagonal symmetry as shown in Figs. 3(a) and 3(b).

To further characterize the Skyrmion crystals, we introduce the local density of Skyrmions $\chi \mathbf{i}$ at lattice site $\mathbf{i}$ in each $x y$ plane as $[45,50]$

$$
\chi \mathbf{i}=\frac{1}{8 \pi}\left[\vec{S}_{\mathbf{i}} \cdot\left(\vec{S}_{\mathbf{i}+\hat{x}} \times \vec{S}_{\mathbf{i}+\hat{y}}\right)+\vec{S}_{\mathbf{i}} \cdot\left(\vec{S}_{\mathbf{i}-\hat{x}} \times \vec{S}_{\mathbf{i}-\hat{y}}\right)\right],
$$

which is the discretization counterpart of the well-known topological charge density $\vec{S} \cdot\left(\partial_{x} \vec{S} \times \partial_{y} \vec{S}\right) / 4 \pi$ for the continuum case [36]. For a single localized 2D Skyrmion here, its topological winding number given by $W_{2 \mathrm{D}}=\sum_{\mathbf{i}}^{\text {unit cell }} \chi \mathbf{i}$ plus the sign of its pole (i.e., here $S_{\mathbf{i}}^{z}=-1$ at the Skyrmion cone as shown in Fig. 3) is equal to a unit in the continuum limit. Note that for an ordinary vortex, this topological number is equal to zero. The winding number is stable with respect to the discretization, as for a lattice layer with size $L \times L$, the fluctuation (error) is on the order of $O\left(4 \pi^{2} / L^{2}\right)$ [50]. In Figs. 3(e) and 3(f), we show local density of the square and the hexagonal Skyrmion crystals. The winding number of a single Skyrmion in the two cases is numerically computed to be nearly -1 .

\section{Interlayer spin interactions and why no 3D Skyrmions}

Finally in this section, we consider the interlayer spin-spin interactions along the $\hat{z}$ axis and check the stability of the square and hexagonal Skyrmion crystals obtained previously [see Figs. 3(a) and 3(b)] with respect to the parameters $\lambda_{1}$ and $\lambda_{2}$. Our numerical results are shown in Fig. 4. We find that the Skyrmion crystals with both square and hexagonal structures are stable and have a large region in the parameter space $\left(\lambda_{1}, \lambda_{2}\right)$. Since the parameters $\lambda_{1}$ and $\lambda_{2}$ are position independent, the spin configurations still exhibit the same distribution of Skyrmions in each $x y$ layer, which has been confirmed in our numerical calculations. That is to say, the 2D Skyrmion crystal states extend along the $\hat{z}$ axis in a columnar manner [46]. In the parameter space (see Fig. 4), there is another phase, i.e., the ferromagnetic phase. This demonstrates that the layer Skyrmion crystals can be melted into the conventional ferromagnet by spin-spin interactions along the $\hat{z}$ axis in Hamiltonian (8). We note that near the boundary between the two phases, the Skyrmions and ferromagnet coexist in the spin configurations and hence the Skyrmion crystals are ill-defined there. However, in most of the region denoted by SKX in Figs. 4(a) and 4(b), the square and hexagonal Skyrmion crystals remain.

In our numerical calculations done by the classical MC method, we do not find a topologically nontrivial spin structure forming a genuine 3D Skyrmion or its corresponding crystal state [51] for the spin Hamiltonian (8) in a large parameter region. For the most isotropic cases with $\theta_{\eta}=\theta=\pi / 4$ and
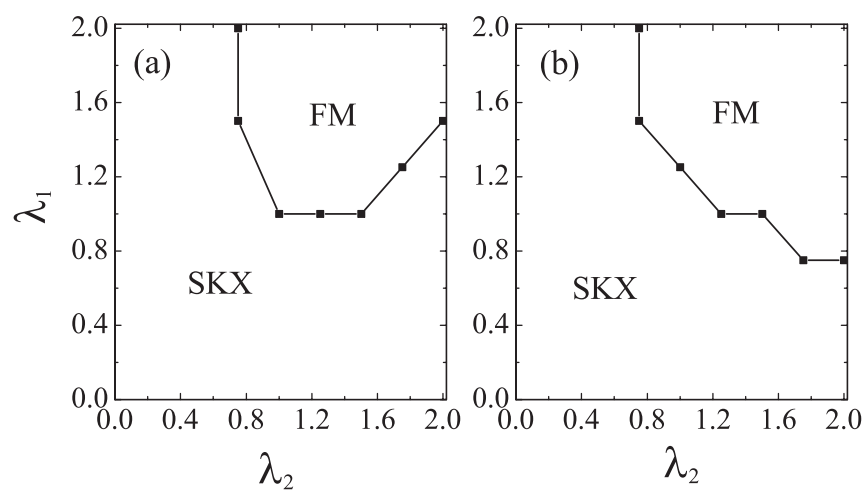

FIG. 4. Stability of layer Skyrmion crystals with respect to the spin interaction parameters $\lambda_{1}$ and $\lambda_{2}$ along the $\hat{z}$ axis. The parameter regions for (a) square and (b) hexagonal Skyrmion crystals denoted by SKX are both wide. Another region denoted by FM is the ferromagnetic phase. The parameters are $\theta=0.726$ and $h_{z}=0.910$ in (a); $\theta=0.592$ and $h_{z}=0.756$ in (b). 
$h_{z}=0$ (one has $D_{\eta}=K_{\eta}=1$ and $J_{\eta}=0$ ), the classical ground state is a spiral state with four-site periodicity along one axis $(\hat{x}, \hat{y}$, or $\hat{z}$ axis). An additional small Zeeman field favors a certain direction. Note that another degenerate spiral state with six-site periodicity in the [111] direction do not appear in the numerical simulations, probably owing to the finite size effects. For other values of $\theta$ with $h_{z}=0$, we find similar behavior for the ground states, i.e., the spins in each plane aligning in the same pattern and extending along the perpendicular axis in a columnar manner. At a strong Zeeman field, all these spins states would be melted into a ferromagnetic state along the direction of the Zeeman field. We have also checked that the special Zeeman field along the [111] direction has similar effects as those along other directions, such as modulating the polarization of the spiral spins and changing the density of the 2D Skymions, without bringing the 3D Skyrmions.

Similar 3D spin models in the context of chiral magnetism have been investigated by variational energy analysis within the global-spin constraint condition $[45,46]$. Detailed calculations in Ref. [45] indicate that a 3D Skyrmionic ground state is possible; however, there would be other 3D spin states with lower energy as shown in Ref. [46]. So, whether 3D Skyrmion crystal states can be energetically stable is yet to be explored in spin models [52]. Returning to our numerical results, there is no indication of such a classical state at low temperature. This is consistent with a very recent numerical work by the classical MC simulation for a similar 3D lattice spin model [53]. The discrepancy might also be due to the soft spins constraint $\left\langle\vec{S}_{\mathbf{i}}^{2}\right\rangle=1$ used in the variational calculations in Refs. $[45,46]$, which replaces the hard spins constraint $\vec{S}_{\mathbf{i}}^{2}=1$ in the MC simulation. We note that this cold atom system with tunable parameters may provide a better platform for exploring the Skyrmion physics and searching for 3D Skyrmion crystal states in a semiclassical (such as using the soft spins) or quantum approach, which would be an interesting challenge in our further studies.

\section{DISCUSSION AND CONCLUSION}

Before concluding this paper, we briefly discuss some methods for detecting the superfluidity states and the spin configurations in the deep MI phase. The plane-wave and the strip superfluidity phases correspond to BECs at a single finite momentum and at a pair of opposite momenta, respectively. The values of the momenta depend on the SO-coupling strength as discussed in Sec. III. The standard time-of-flight imaging measurement can reveal the condensate peaks at the nontrivial momentum points [26], which provides direct signatures of the two superfluidity states. The different magnetic orders presented above can be detected by the optical Bragg scattering for atoms in OLs [54], as the peaks in the Bragg spectroscopy directly reveal their spin structure factors. Another way to measure the spin configurations is using the spin-resolved in situ imaging technique [55]. The idea is to implement a high-resolution optical imaging system, by which single atoms are detected with near-unity fidelity on individual sites of an OL [55]. A similar measurement has been performed for revealing phase transitions of an atomic Ising chain [56].

In summary, we have studied the superfluidity and magnetic properties of ultracold bosons with the synthetic 3D SO coupling in a cubic OL. The lowest energy band exhibits one, two, or four pairs of degenerate single-particle ground states depending on the SO-coupling strengths, which can lead to the condensate states with spin stripes for the weak atomic interactions. In the MI state with one particle per site, an effective spin Hamiltonian with 3D DM spin interactions is derived. The spin Hamiltonian with an additional Zeeman field has a rich phase diagram with spiral, stripe, vortex-crystal, and Skyrmion crystal spin textures in each $x y$-plane layer. The Skyrmion crystals extended along the $z$ axis in a columnar manner can be tunable with square and hexagonal symmetries, and stable against the interlayer spin-spin interactions in a large parameter region.

\section{ACKNOWLEDGMENTS}

We thank Tao Zhou and Yan Chen for helpful discussions. This work was supported by the NSFC (Grant No. 11125417), the SKPBR (Grant No. 2011CB922104), the PCSIRT, the SRFGS of SCNU, the GRF (Grants No. HKU7058/11P and No. HKU7045/13P) and CRF (Grant No. HKU-8/11G) of the RGC of Hong Kong, and the URC fund of HKU.
[1] X.-L. Qi and S.-C. Zhang, Phys. Today 63(1), 33 (2010).

[2] M. Z. Hasan and C. L. Kane, Rev. Mod. Phys. 82, 3045 (2010).

[3] For a review, see J. Dalibard, F. Gerbier, G. Juzeliūnas, and P. Öhberg, Rev. Mod. Phys. 83, 1523 (2011), and references therein.

[4] S. L. Zhu, H. Fu, C. J. Wu, S. C. Zhang, and L. M. Duan, Phys. Rev. Lett. 97, 240401 (2006); D. W. Zhang, Z. Y. Xue, H. Yan, Z. D. Wang, and S. L. Zhu, Phys. Rev. A 85, 013628 (2012); D. W. Zhang, L.-B. Shao, Z. Y. Xue, H. Yan, Z. D. Wang, and S. L. Zhu, ibid. 86, 063616 (2012).

[5] Y.-J. Lin, K. Jiménez-García, and I. B. Spielman, Nature (London) 471, 83 (2011).
[6] J.-Y. Zhang, S.-C. Ji, Z. Chen, L. Zhang, Z.-D. Du, B. Yan, G.-S. Pan, B. Zhao, Y.-J. Deng, H. Zhai, S. Chen, and J.-W. Pan, Phys. Rev. Lett. 109, 115301 (2012).

[7] P. Wang, Z.-Q. Yu, Z. Fu, J. Miao, L. Huang, S. Chai, H. Zhai, and J. Zhang, Phys. Rev. Lett. 109, 095301 (2012).

[8] L. W. Cheuk, A. T. Sommer, Z. Hadzibabic, T. Yefsah, W. S. Bakr, and M. W. Zwierlein, Phys. Rev. Lett. 109, 095302 (2012).

[9] For a review, see H. Zhai, Int. J. Mod. Phys. B 26, 1230001 (2012), and references therein.

[10] C. Wang, C. Gao, C. M. Jian, and H. Zhai, Phys. Rev. Lett. 105, 160403 (2010).

[11] C. Wu, I. Mondragon-Shem, C. Wu, I. Mondragon-Shem, and X. F. Zhou, Chin. Phys. Lett. 28, 097102 (2011). 
[12] S. Sinha, R. Nath, and L. Santos, Phys. Rev. Lett. 107, 270401 (2011).

[13] H. Hu, B. Ramachandhran, H. Pu, and X. J. Liu, Phys. Rev. Lett. 108, 010402 (2012).

[14] J. Larson, J.-P. Martikainen, A. Collin, and E. Sjöqvist, Phys. Rev. A 82, 043620 (2010).

[15] T. Graß, K. Saha, K. Sengupta, and M. Lewenstein, Phys. Rev. A 84, 053632 (2011).

[16] W. S. Cole, S. Zhang, A. Paramekanti, and N. Trivedi, Phys. Rev. Lett. 109, 085302 (2012).

[17] J. Radić, A. Di Ciolo, K. Sun, and V. Galitski, Phys. Rev. Lett. 109, 085303 (2012).

[18] M. Gong, Y. Qian, V. W. Scarola, and C. Zhang, arXiv:1205.6211.

[19] Z. Cai, X. Zhou, and C. Wu, Phys. Rev. A 85, 061605(R) (2012).

[20] S. Mandal, K. Saha, and K. Sengupta, Phys. Rev. B 86, 155101 (2012).

[21] J.-P. Martikainen, Phys. Rev. A 86, 033630 (2012).

[22] W. Han, S. Zhang, and W.-M. Liu, arXiv:1211.2097.

[23] C. H. Wong and R. A. Duine, Phys. Rev. Lett. 110, 115301 (2013).

[24] M. P. A. Fisher, P. B. Weichman, G. Grinstein, and D. S. Fisher, Phys. Rev. B 40, 546 (1989).

[25] D. Jaksch, C. Bruder, J. I. Cirac, C. W. Gardiner, and P. Zoller, Phys. Rev. Lett. 81, 3108 (1998).

[26] M. Greiner, O. Mandel, T. Esslinger, T. W. Hansch, and I. Bloch, Nature (London) 415, 39 (2002).

[27] I. E. Dzyaloshinskii, J. Phys. Chem. Solids 4, 241 (1958); T. Moriya, Phys. Rev. 120, 91 (1960).

[28] B. M. Anderson, G. Juzeliūnas, V. M. Galitski, and I. B. Spielman, Phys. Rev. Lett. 108, 235301 (2012).

[29] Y. Li, X. Zhou, and C. Wu, Phys. Rev. B 85, 125122 (2012).

[30] D. Jaksch and P. Zoller, New J. Phys. 5, 56 (2003); F. Gerbier and J. Dalibard, ibid. 12, 033007 (2010).

[31] M. Yang and S. L. Zhu, Phys. Rev. A 82, 064102 (2010).

[32] A. Bermudez, L. Mazza, M. Rizzi, N. Goldman, M. Lewenstein, and M. A. Martin-Delgado, Phys. Rev. Lett. 105, 190404 (2010).

[33] L. Balents, Physics 4, 36 (2011).

[34] Q. Han, J. Liu, D.-B. Zhang, and Z. D. Wang, arXiv:1104.0614.

[35] J. P. Vyasanakere and V. B. Shenoy, Phys. Rev. B 83, 094515 (2011); J. P. Vyasanakere, S. Zhang, and V. B. Shenoy, ibid. 84, 014512 (2011); L. He and X.-G. Huang, ibid. 86, 014511 (2012); X. Cui, Phys. Rev. A 85, 022705 (2012).

[36] T. Kawakami, T. Mizushima, M. Nitta, and K. Machida, Phys. Rev. Lett. 109, 015301 (2012).
[37] Y. Li, X. Zhou, and C. Wu, arXiv:1205.2162.

[38] B. M. Anderson and C. W. Clark, J. Phys. B: At. Mol. Opt. Phys. 46, 134003 (2013).

[39] U. A. Khawaja and H. T. C. Stoof, Nature (London) 411, 918 (2001); J. Ruostekoski and J. R. Anglin, Phys. Rev. Lett. 86, 3934 (2001); R. A. Battye, N. R. Cooper, and P. M. Sutcliffe, ibid. 88, 080401 (2002); A. Tokuno, Y. Mitamura, M. Oshikawa, and I. F. Herbut, Phys. Rev. A 79, 053626 (2009).

[40] R. Barnett, S. Powell, T. Graß, M. Lewenstein, and S. Das Sarma, Phys. Rev. A 85, 023615 (2012).

[41] A. Auerbach, Interacting Electrons and Quantum Magnetism (Springer-Verlag, Berlin, 1998).

[42] A. B. Kuklov and B. V. Svistunov, Phys. Rev. Lett. 90, 100401 (2003).

[43] L.-M. Duan, E. Demler, and M. D. Lukin, Phys. Rev. Lett. 91, 090402 (2003).

[44] S. L. Zhu, L.-B. Shao, Z. D. Wang, and L.-M. Duan, Phys. Rev. Lett. 106, 100404 (2011); C. Zhang, Phys. Rev. A 82, $021607(\mathrm{R})$ (2010).

[45] S. D. Yi, S. Onoda, N. Nagaosa, and J. H. Han, Phys. Rev. B 80, 054416 (2009).

[46] J.-H. Park and J. H. Han, Phys. Rev. B 83, 184406 (2011).

[47] U. K. Rößler, A. N. Bogdanov, and C. Pfleiderer, Nature (London) 442, 797 (2006).

[48] X. Z. Yu, Y. Onose, N. Kanazawa, J. H. Park, J. H. Han, Y. Matsui, N. Nagaosa, and Y. Tokura, Nature (London) 465, 901 (2010).

[49] J. M. Thijssen, Computational Physics (Cambridge University Press, Cambridge, 2007).

[50] E. Alba, X. Fernandez-Gonzalvo, J. Mur-Petit, J. K. Pachos, and J. J. Garcia-Ripoll, Phys. Rev. Lett. 107, 235301 (2011).

[51] I. R. Klebanov, Nucl. Phys. B 262, 133 (1985); M. Kugler and S. Shtrikman, Phys. Rev. D 40, 3421 (1989).

[52] F. N. Rybakov, A. B. Borisov, and A. N. Bogdanov, Phys. Rev. B 87, 094424 (2013). The 3D modulated Skyrmionic ground states in these works are not the genuine 3D Skyrmionic states, since they carry a zero 3D topological winding number.

[53] S. Buhrandt and L. Fritz, arXiv:1304.6580.

[54] T. A. Corcovilos, S. K. Baur, J. M. Hitchcock, E. J. Mueller, and R. G. Hulet, Phys. Rev. A 81, 013415 (2010).

[55] W. S. Bakr, J. I. Gillen, A. Peng, S. Fölling, and M. Greiner, Nature (London) 462, 74 (2009); N. Gemelke, X. Zhang, C.-L. Hung, and C. Chin, ibid. 460, 995 (2009).

[56] J. Simon, W. S. Bakr, R. Ma, M. E. Tai, P. M. Preiss, and M. Greiner, Nature (London) 472, 307 (2011). 